\title{
Evolutions of matter-wave bright soliton with spatially modulated nonlinearity
}

\author{
Yongshan Cheng (程永山) $)^{1,2 *}$ and Fei Liu (刘 飞) $)^{2}$ \\ ${ }^{1}$ Instituto de Física Teórica, Universidade Estadual Paulista, São Paulo 01.405-900, São Paulo, Brazil \\ ${ }^{2}$ Department of Physics, Hubei Normal University, Huangshi 435002, China \\ *E-mail: yong_shan@163.com
}

Received December 8, 2008

\begin{abstract}
The evolution characteristics of a matter-wave bright soliton are investigated by means of the variational approach in the presence of spatially varying nonlinearity. It is found that the atom density envelope of the soliton is changed as a result of the spatial variation of the s-wave scattering length. The stable soliton can exist in appropriate initial conditions. The movement of the soliton depends on the sign and value of the coefficient of spatially modulated nonlinearity. These theoretical predictions are confirmed by the full numerical simulations of the one-dimensional Gross-Pitaevskii equation.

OCIS codes: 190.4720, 270.5530, 140.7010.
\end{abstract}

doi: 10.3788/COL20090709.0834.

At sufficiently low temperatures, the mean-field description of the Bose-Einstein condensate (BEC) dynamics is based on the nonlinear Schrödinger equation (NLSE) with the trapping potential, also called GrossPitaevskii equation, for the mean-field wave function $\psi$ in three-dimensions ${ }^{[1]}$. $\psi$ is normalized to the number $N^{\prime}=\int|\psi|^{2} \mathrm{~d} r$ of condensed atoms. In the realistic experimental setting, an external electromagnetic field is used to produce, trap, and manipulate the BEC. Experiments with BECs have now acquired a high degree of control over the key parameters of the system. Using a strongly anisotropic trapping potential, it is possible to freeze the atomic motion along one or several directions; therefore, one realizes in this way the BEC with reduced dimensionality ${ }^{[2,3]}$. Using Feshbach resonances driven by a magnetic ${ }^{[4]}$ or optical ${ }^{[5]}$ field, one can also adjust almost at will the interaction strength and sign between the atoms. Recently, the dynamics of matter-wave solitons with different types of management of system parameters has been under intensive investigations. Two types of modulations have been considered: dispersion and nonlinearity management, which can occur both in time and in space. In nonlinearity modulation, Fedichev et al. predicted that the spatial variation of the laser field intensity by proper choice of the resonance detuning could lead to the spatial dependence of the atomic scattering length ${ }^{[6]}$, i.e., so-called "collisionally inhomogeneous" BECs. The theoretical result has been demonstrated in recent experiment with the ${ }^{87} \mathrm{Rb}$ condensate ${ }^{[5]}$. In the experiment, atoms in a ${ }^{87} \mathrm{Rb}$ condensate are exposed to laser light which is tuned close to the transition frequency to an excited molecular state. By controlling the power and detuning of the laser beam the atomic scattering length is changed over one order of magnitude, from $10 a_{0}$ to $190 a_{0}$ ( $a_{0}$ is the Bohr radius). It is well known that the nonlinearity term in the NLSE is introduced by the inter-atomic interactions, which are taken into regard through an effective mean field. The coefficient of the nonlinear term is controlled by the s-wave scattering length $a_{\mathrm{s}}$, whose sign and magnitude determine many of the fundamental properties of BECs such as their shape or collective excitations. Motivated by the experiment, a lot of theoretical work has been carried out related to the existence and stability ${ }^{[7,8]}$, dynamical trapping and transmission ${ }^{[9,10]}$, and dissipative dynamics ${ }^{[11]}$ of matter-wave solitons in a collisionally inhomogeneous environment. Despite the aforementioned experimental and theoretical work, the evolution characteristics of a BEC bright soliton with spatially modulated nonlinearity have so far not been analyzed thoroughly. The aim of the present work is to investigate the evolution characteristics of a BEC bright soliton trapped in a nonlinear optical lattice (OL) by the variational approach. With the help of the idea of an effective potential ${ }^{[12]}$, the motion of the soliton's center-of-mass is discussed.

We adopt the trapping potential that is a weak OL along the longitudinal direction ( $x$ direction) and a strong harmonic magnetic field in the radial directions $(y, z$ directions). The dynamics for a one-dimensional (1D) mean-field wave function $u \equiv u(x, t)$ can be described by ${ }^{[13]}$

$$
\mathrm{i} \hbar \frac{\partial u}{\partial t}=-\frac{\hbar^{2}}{2 m} \frac{\partial^{2} u}{\partial x^{2}}+g_{1 D}|u|^{2} u+V(x) u,
$$

where $V(x)=2 V_{0} \cos ^{2}(k x)$ is the OL potential which is created by two counter propagating laser beams near the optical induced Feshbach resonance, $V_{0}$ is the lattice depth and the lattice wave number $k$ can be tuned by the geometry of laser beams. In the case of the long-period OL, $k$ is small so that the OL is smooth and slowly varying on the soliton scale. $g_{1 D}=2 \hbar^{2} a_{\mathrm{s}} / m a_{\perp}^{2}, a_{\perp}=\left(\hbar / m \omega_{\perp}\right)^{1 / 2}$ is the transverse harmonic-oscillator length, $\omega_{\perp}$ is the transverse confinement frequency, and $m$ is the mass of an atom inside the BEC. The $s$-wave scattering length $a_{\mathrm{s}}$ accounts for the repulsive $\left(a_{\mathrm{s}}>0\right)$ and attractive $\left(a_{\mathrm{s}}<0\right)$ inter-atomic interactions respectively. In this letter, we only investigate the bright matter-wave soliton in the presence of a spatially varying nonlinearity, which exists in the case of attraction. Assuming that this condensate is collisionally inhomogeneous, as explained above, the 
spatial variation of laser field intensity $I(x)=I_{0} \cos ^{2}(k x)$ produces the spatial variation of the atomic scattering length $a_{\mathrm{s}}=a_{\mathrm{s} 0}+a_{\mathrm{s} 1} \cos ^{2}(k x)$, where $a_{\mathrm{s} 0}$ is the s-wave scattering length in the absence of the external trapping potential and is a negative value corresponding to attractive condensates, $a_{\mathrm{s} 1}$ is a constant related to the optical intensity and may be either positive or negative. Then, $u(x, t)$ satisfies the following normalized 1D NLSE:

$$
\begin{aligned}
\mathrm{i} \frac{\partial u}{\partial t} & +\frac{1}{2} \frac{\partial^{2} u}{\partial x^{2}}+\left[\varepsilon_{0}+\varepsilon_{1} \cos (2 k x)\right]|u|^{2} u \\
& =V_{0} \cos (2 k x) u .
\end{aligned}
$$

In Eq. (2), we have introduced the dimensionless variables $t=t^{\prime} / \omega_{\perp}, x=x^{\prime} a_{\perp}, u=u^{\prime} / a_{\perp}^{1 / 2}$, and dropped the primes on rescaled variables to simplify the notation. $\varepsilon_{0}$ is defined as $\varepsilon_{0}=2 a_{\mathrm{s} 0} / a_{\perp}+\varepsilon_{1}$, where $\varepsilon_{1}=a_{\mathrm{s} 1} / a_{\perp}$ is the spatially modulated coefficient and is small. $V_{0}$ is measured in the unit of $\hbar \omega_{\perp}$, and an additive constant $V_{0}$ has been omitted because it does not affect the dynamics of the BEC. $a_{\mathrm{s} 0}$ is presented in form of the absolute value. The number of particles in the $1 \mathrm{D}$ system (2), defined as $N=\int_{-\infty}^{+\infty}|u|^{2} \mathrm{~d} x$, has a relation to the actual number of atoms $N^{\prime}$ as $N^{\prime}=N / a_{\perp}$, and $N$ is approximately $10^{0}-10^{1[12]}$. Below we will use only the quantity $N$ referring to it as "the number of atoms" for simplicity.

If $\varepsilon_{0}=1, \varepsilon_{1}=0$, and $V_{0}=0$, Eq. (2) is a standard NLSE that possesses an exact bright soliton solution. In the case that Eq. (2) is supplemented by the external small perturbation, i.e., $V_{0}$ and $\varepsilon_{1}$ are both of small value, however, the shifts of the bright soliton's parameters take place during the evolution process. As we are interested in the evolution of the BEC bright soliton, this issue can be addressed by using the variational approach. The standard variational approach is a procedure for obtaining approximate solutions of the NLSE when a trial solution containing unknown parameters is assumed. The main advantage of the approach is that it provides explicit, although approximate, analytical expressions for the parameters. These analytical expressions give some of the most important features of the solution and provide us with a better physical understanding of the soliton. The main shortcoming of the use of trial solution is the inability to account for changes in pulse shape ${ }^{[14-16]}$. We adopt the Gaussian function (quasi-soliton) below as the trial function (variational ansatz):

$u(x, t)=A \exp \left[-B^{2}\left(x-x_{0}\right)^{2} / 2\right] \exp \left\{\mathrm{i}\left[C\left(x-x_{0}\right)+\phi\right]\right\}$,

where $A, B$, and $x_{0}$ represent the amplitude, inverse width in space, and location of the soliton's center-ofmass, respectively; $\phi$ is the phase; $C$ defines both the linear phase coefficient and the wave number. It is important to emphasize that the object that we are going to study is not the soliton in the mathematical meaning, but rather "a solitary wave" as it appears in a nonintegrable model. On the other hand, $A$ and $B$ are mutually independent because the perturbation terms have been introduced. During the evolution of the soliton, $u(x, t)$ retains the Gaussian shape given by Eq. (3), but the parameters are the functions of time as a result of the small perturbation.

We obtain the averaged Lagrangian of Eq. (2) by the ansatz (3):

$$
\begin{aligned}
L(t)= & \int_{-\infty}^{\infty}\left\{\frac{\mathrm{i}}{2}\left(u^{*} \frac{\partial u}{\partial t}-u \frac{\partial u^{*}}{\partial t}\right)-\frac{1}{2}\left|\frac{\partial u}{\partial x}\right|^{2}+\frac{1}{2}\left[\varepsilon_{0}+\varepsilon_{1} \cos (2 k x)\right]|u|^{4}-V_{0} \cos (2 k x)|u|^{2}\right\} \mathrm{d} x \\
= & \sqrt{\pi} \frac{A^{2}}{B}\left(C \dot{x}_{0}-\dot{\phi}\right)-\frac{\sqrt{\pi}}{2} \frac{A^{2}}{B}\left(\frac{B^{2}}{2}+C^{2}\right)+\frac{\sqrt{\pi}}{2 \sqrt{2}} \frac{A^{4}}{B}\left[\varepsilon_{0}+\varepsilon_{1} \cos \left(2 k x_{0}\right) \exp \left(-\frac{k^{2}}{2 B^{2}}\right)\right] \\
& -\sqrt{\pi} V_{0} \frac{A^{2}}{B} \cos \left(2 k x_{0}\right) \exp \left(-\frac{k^{2}}{B^{2}}\right),
\end{aligned}
$$

where the dot over the parameters denotes a differential with respect to time $t$ and the asterisk denotes the complex conjugate. Using the Euler-Lagrange equations, $\frac{\partial L}{\partial \sigma}-\frac{\mathrm{d}}{\mathrm{d} t}\left(\frac{\partial L}{\partial \dot{\sigma}}\right)=0$, the following equations are obtained when selecting $\sigma=\phi, x_{0}, C, A$, and $B$ :

$$
\begin{aligned}
& \sqrt{\pi} A^{2} / B=\text { constant } \equiv N, \\
& \dot{C}=-\frac{\varepsilon_{1} k}{\sqrt{2 \pi}} N B \sin \left(2 k x_{0}\right) \exp \left(-\frac{k^{2}}{2 B^{2}}\right)+2 k V_{0} \sin \left(2 k x_{0}\right) \exp \left(-\frac{k^{2}}{B^{2}}\right), \\
& \dot{x}_{0}=C, \\
& -B^{2}+\frac{N B}{\sqrt{2 \pi}}\left[\varepsilon_{0}+\varepsilon_{1} \cos \left(2 k x_{0}\right)\left(\frac{k^{2}}{B^{2}}+1\right) \exp \left(-\frac{k^{2}}{2 B^{2}}\right)\right]-V_{0} \frac{4 k^{2}}{B^{2}} \cos \left(2 k x_{0}\right) \exp \left(-\frac{k^{2}}{B^{2}}\right)=0, \\
& \dot{\phi}=\frac{C^{2}}{2}+\frac{N B}{4 \sqrt{2 \pi}}\left[3 \varepsilon_{0}-\varepsilon_{1} \cos \left(2 k x_{0}\right)\left(\frac{k^{2}}{B^{2}}-3\right) \exp \left(-\frac{k^{2}}{2 B^{2}}\right)\right]-V_{0}\left(1-\frac{k^{2}}{B^{2}}\right) \cos \left(2 k x_{0}\right) \exp \left(-\frac{k^{2}}{B^{2}}\right) .
\end{aligned}
$$


Equations (5a)-(5e) determine the evolution of the BEC bright soliton. It is clear that there are terms in these equations that are explicitly dependent on $\varepsilon_{1}$ describing the effects predicted when the spatial variation of the s-wave scattering length is taken into account. In the case of $\varepsilon_{1}=0$ and $V_{0}=0$, we can conclude that the BEC bright soliton moves with a constant speed and a stable shape.

It is shown that the phase has no effect on the velocity, width, and wave number of the soliton. On the contrary, according to Eq. (5e), the phase is determined by other parameters during the evolution of the BEC soliton. We are primarily interested in both the evolution of the atom density envelope and the movement behavior of the BEC soliton and, hence, we leave Eq. (5e) and discuss the other equations.

Equation (5a) can be interpreted as a conservation law (the conservation of the atom number) which is related to the normalization of the trial function and $N=\int_{-\infty}^{+\infty}|u|^{2} \mathrm{~d} x=\sqrt{\pi} A^{2} / B$ is a constant. Although we infer from Eq. (5a) that $A^{2} / B$ is still a constant in presence of the spatially modulated nonlinearity, Eq. (5d) can be regarded as an equation determining $A$ and $B$ once the other parameters are known so that the equations are self-consistent. Therefore, Eq. (5d) is the constraint equation of the parameters for a stable BEC soliton. It shows that $B$ is related to the spatially modulated coefficient $\varepsilon_{1}$. To understand the effects, in Fig. 1, we plot the inverse width of the BEC soliton versus the spatially modulated coefficient $\varepsilon_{1}$ according to Eq. (5d) when the BEC soliton is stable. It shows that the inverse width of a stable BEC soliton increases as $\varepsilon_{1}$ increases. This is because for positive $\varepsilon_{1}$ the nonlinearity becomes stronger, leading to the narrower width . In the opposite case $\left(\varepsilon_{1}<0\right)$, the nonlinearity is weaker and the soliton's width has to increase. When the nonlinearity is weak enough, for example, when $-0.5<\varepsilon_{1}<-0.3$, the effects of nonlinearity on the width is small, as shown in the figure. Then the width is primarily determined by the dispersion and the trapping potential.

Equation (5c) is the equation determining the movement of the soliton's center-of-mass. It shows that the modification brought about by the spatially modulated nonlinearity is to alter the velocity of the soliton's center-of-mass. In order to investigate the effects of the

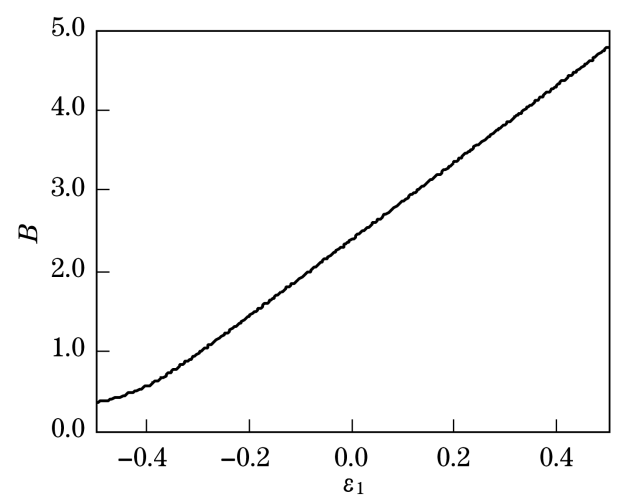

Fig. 1. Inverse width of the BEC soliton versus the spatially modulated coefficient $\varepsilon_{1}$. The parameters are selected as $N=6, k=0.1$ (the long-period OL), $2 a_{\mathrm{s} 0} / a_{\perp}=1, x_{0}=0$, and $V_{0}=-0.5$. spatially modulated nonlinearity on the soliton's movement, we insert Eq. (5b) into Eq. (5c). An anharmonic effective potential is derived using the classical mechanics analogy, which is

$$
\begin{aligned}
V_{\text {eff }}= & {\left[-\frac{\varepsilon_{1} N B}{2 \sqrt{2 \pi}} \exp \left(-\frac{k^{2}}{2 B^{2}}\right)+V_{0} \exp \left(-\frac{k^{2}}{B^{2}}\right)\right] } \\
& \times \cos \left(2 k x_{0}\right) \equiv V_{\text {eff } 1}+V_{\text {eff } 2 .}
\end{aligned}
$$

where $V_{\text {eff2 }}=V_{0} \exp \left(-k^{2} / B^{2}\right) \cos \left(2 k x_{0}\right)$ arises from the OL. We find that the effective potential is not equal to the external trapping potential and is related to the parameters of the soliton. $V_{\text {eff1 }}=$ $-\frac{\varepsilon_{1} N B}{2 \sqrt{2 \pi}} \exp \left(-k^{2} /\left(2 B^{2}\right)\right) \cos \left(2 k x_{0}\right)$ is induced by the spatial modulation of the nonlinearity and is actually a pseudo-potential as pointed out in Ref. [17]. The soliton can "feel" the effective potential although it is not a true external trapping potential. Notice that it is a dynamic potential and depends on the soliton characteristics (i.e., $N$ and $B)$. Because of the periodic OL and spatially periodic modulation of the nonlinearity, the entire effective potential $V_{\text {eff }}$ is periodic. By considering the model combining the spatially periodic modulation of the nonlinearity and periodic potential, the similar conclusions are also predicted in Ref. [18] by using the Hamiltonian perturbation approach. Those conclusions provide a quantitative basis for understanding the dynamics of the BEC soliton with spatially modulated nonlinearity. According to Eq. (6), the motion of the BEC bright soliton can be approximately regarded as that of a particle inside an effective potential energy $V_{\text {eff }}$. Thus, we can determine the motion trajectory of the equivalent single particle. By numerical simulations, $V_{\text {eff }}$ versus $x_{0}$ is plotted in Fig. 2 which indicates that the spatially modulated nonlinearity deforms the shape of the effective potential and changes the kinematics characteristics of the soliton depending on the sign and value of $\varepsilon_{1}$. The same parameters as those in Fig. 1 are selected, but $B$ is determined by Eq. (5d). We find that $V_{\text {eff1 }}$ compensates $V_{\text {eff2 }}$, and that $V_{\text {eff }}$ is a potential well which is not equal to the external trapping potential. If we initialize a motionless BEC soliton at the nonequilibrium location, i.e., $x_{0} \neq 0$, it will vibrate around the stable equilibrium point $x=0$. When $\varepsilon_{1}>0, V_{\text {eff }}$ becomes deeper, and the vibration of the BEC soliton should be faster. In the opposite case, when $\varepsilon_{1}<0, V_{\text {eff }}$ becomes shallower, the

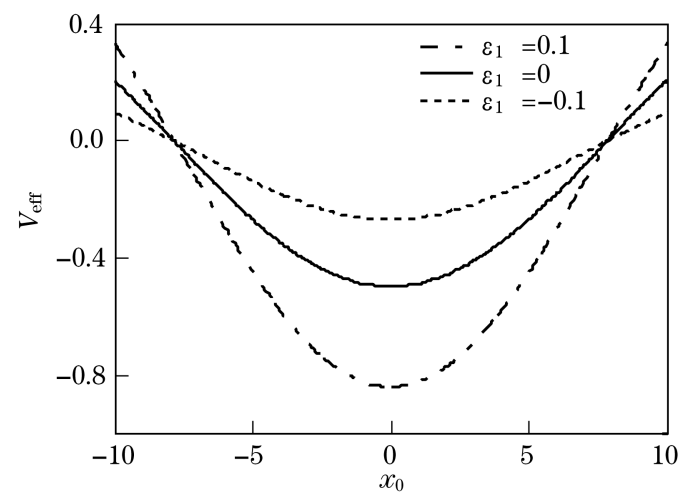

Fig. 2. Effective potential energy $V_{\text {eff }}$ versus location of the soliton's center-of-mass $x_{0} . \quad N=6, k=0.1$, and $V_{0}=-0.5$. B is determined by Eq. (5d). 


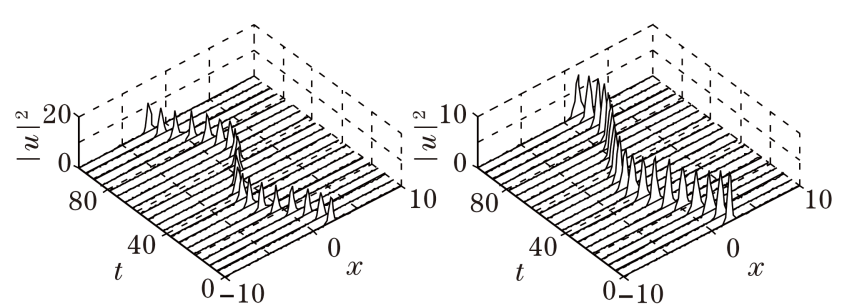

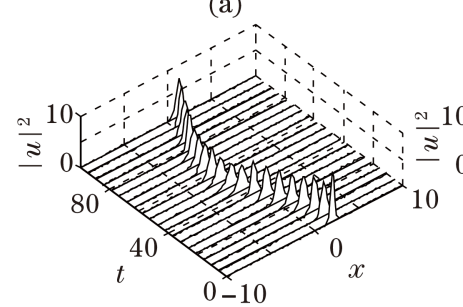

(c) (b)

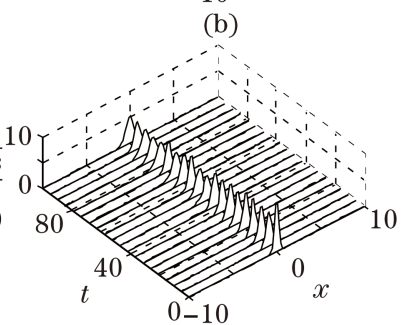

(d)
Fig. 3. Evolution of the atom density envelope of the BEC bright soliton. $x_{0}=2$ and $\varepsilon_{1}=0.1,0,-0.1$ for $(\mathrm{a}),(\mathrm{b}),(\mathrm{c})$, respectively. $x_{0}=0$ and $\varepsilon_{1}=-0.1$ for (d).

vibration should become slower.

In order to make sure of the validity of the theoretical predictions, in Fig. 3, we show the motion of the BEC bright soliton by a direct numerical integration of Eq. (2). The figures indicate the evolution of the BEC soliton's atom density envelope $|u|^{2}$. In our numerical simulations, the initial input pulse is $u(x, t=0)=\left(N B / \pi^{1 / 2}\right)^{1 / 2} \exp \left[-B^{2}\left(x-x_{0}\right)^{2} / 2\right]$ where $N=6, B=3$, and $x_{0}=2$. The nonlinear coefficients are $2 a_{\mathrm{s} 0} / a_{\perp}=1$, and $\varepsilon_{1}=0.1,0,-0.1$ for Figs. 3(a), (b), (c), respectively. The parameters of the OL are $k=0.1$ and $V_{0}=-0.5$. We can find that the soliton vibrates in the periodic manner around the point $x=0$. Compared with Fig. 3(b) where $\varepsilon_{1}=0$, the vibration of the BEC soliton is faster when $\varepsilon_{1}=0.1$ as shown in Fig. 3(a). On the contrary, Fig. 3(c) indicates clearly that the vibration of the BEC soliton slows down when $\varepsilon_{1}=-0.1$. In these figures, we also find that the soliton's width alternates during vibration. This is because the inverse width of a stable BEC soliton is related to the location of the BEC soliton's center-of-mass as predicted in Fig. 1. When $\varepsilon_{1}=0.1$, however, the alternation is small, and the amplitude is slightly larger. The reason is that $\varepsilon_{0}$ is larger and the nonlinearity is dominant. The effect of the location of the soliton's center-of-mass on the width is small according to Eq. (5d). When $\varepsilon_{1}=-0.1, \varepsilon_{0}$ becomes small and the terms that contain the cosine function are dominant. Then the soliton's width alternates periodically as $x_{0}$ alternates, which is shown in Fig. 3(c). These conclusions are in agreement with the foregoing theoretical predictions. It is important to emphasize that the BEC soliton's width is also related to the stability. When an unstable soliton is introduced, it will evolve into a stable soliton, and the soliton's width will alternate. This is shown in Fig. 3(d) for which the same parameters of the simulation are selected as those in Fig. 3(c) except $x_{0}=0$ in the initial input pulse.

In conclusion, we have investigated the evolution and motion characteristics of a BEC bright soliton when the $s$-wave scattering length is spatially modulated by the OL. It is found that a stable BEC bright soliton may exist in appropriate initial conditions. The coefficient of the spatially modulated nonlinearity plays an important role and determines the soliton's parameters during the evolution of the BEC bright soliton. The spatial modulation of nonlinearity term also changes the movement of the soliton depending on the sign and value of the coefficient of the spatially modulated nonlinearity. According to the investigation, one can understand the evolution characteristics of a BEC bright soliton. In view of the practical applications of the BEC soliton, understanding these properties is important.

This work was supported by the Post-Doctoral Program of FAPESP, São Paulo, Brazil (No. 08/539796 ) and the Science and Technology Program of Education Department of Hubei Province, China (No. D200722003).

\section{References}

1. F. Dalfovo, S. Giorgini, L. P. Pitaevskii, and S. Stringari, Rev. Mod. Phys. 71, 463 (1999)

2. Y. Cheng, H. Li, and R. Gong, Chin. Opt. Lett. 3, 715 (2005).

3. E. A. Donley, N. R. Claussen, S. L. Cornish, J. L. Roberts, E. A. Cornell, and C. E. Wieman, Nature 412, 295 (2001).

4. S. Inouye, M. R. Andrews, J. Stenger, H.-J. Miesner, D. M. Stamper-Kurn, and W. Ketterle, Nature 392, 151 (1998).

5. M. Theis, G. Thalhammer, K. Winkler, M. Hellwig, G. Ruff, R. Grimm, and J. H. Denschlag, Phys. Rev. Lett. 93, 123001 (2004).

6. P. O. Fedichev, Yu. Kagan, G. V. Shlyapnikov, and J. T. M. Walraven, Phys. Rev. Lett. 77, 2913 (1996).

7. H. Sakaguchi and B. A. Malomed, Phys. Rev. E 72, 046610 (2005).

8. A. S. Rodrigues, P. G. Kevrekidis, M. A. Porter, D. J. Frantzeskakis, P. Schmelcher, and A. R. Bishop, Phys. Rev. A 78, 013611 (2008).

9. G. Theocharis, P. Schmelcher, P. G. Kevrekidis, and D. J. Frantzeskakis, Phys. Rev. A 74, 053614 (2006).

10. J. Garnier and F. Kh. Abdullaev, Phys. Rev. A 74, 013604 (2006).

11. F. Kh. Abdullaev, A. Gammal, H. L. F. da Luz, and L. Tomio, Phys. Rev. A 76, 043611 (2007).

12. Y.-S. Cheng, R.-Z. Gong, and H. Li, Chin. Phys. Lett. 24, 35 (2007).

13. L. Salasnich, A. Parola, and L. Reatto, Phys. Rev. A 65, 043614 (2002).

14. B. A. Malomed, Z. H. Wang, P. L. Chu, and G. D. Peng, J. Opt. Soc. Am. B 16, 1197 (1999).

15. H. Li, D. N. Wang, and Y. Cheng, Chin. Opt. Lett. 5, 114 (2007).

16. H. Li and D. Wang, Chin. Opt. Lett. 5, 504 (2007).

17. T. Mayteevarunyoo, B. A. Malomed, and G. Dong, Phys. Rev. A 78, 053601 (2008).

18. Z. Rapti, P. G. Kevrekidis, V. V. Konotop, and C. K. R. T. Jones, J. Phys. A 40, 14151 (2007). 